\title{
Damping and tuning of the fibre violin modes in monolithic silica suspensions
}

\author{
S Goßler ${ }^{1}$, G Cagnoli ${ }^{2}$, D R M Crooks ${ }^{2}$, H Lück ${ }^{1}$, S Rowan $^{2,3}$, \\ J R Smith ${ }^{1}$, K A Strain ${ }^{2}$, J Hough ${ }^{2}$ and K Danzmann ${ }^{1}$ \\ ${ }^{1}$ Max-Planck-Institute for Gravitational Physics (Albert-Einstein-Institute) and University of \\ Hannover, Callinstr 38, D-30167 Hannover, Germany \\ ${ }^{2}$ Department of Physics and Astronomy, Institute for Gravitational Research, University of \\ Glasgow, Glasgow G12 8QQ, UK \\ ${ }^{3}$ Edward L Ginzton Laboratory, Stanford University, Stanford, CA 94305-4088, USA \\ E-mail: sfg@mpq.mpg.de
}

Received 1 September 2003

Published 11 February 2004

Online at stacks.iop.org/CQG/21/S923 (DOI: 10.1088/0264-9381/21/5/082)

\begin{abstract}
High $Q$ mirror suspensions are a key element of the advanced interferometric gravitational-wave detectors. In December 2002 the last of the final interferometer optics of GEO 600 were monolithically suspended, using fused silica fibres. The violin modes of the suspension fibres can have $Q$ greater than $10^{8}$ and can therefore interfere with the interferometer length control servo. Hence, the violin modes need to be damped, without degrading the pendulum $Q$ itself. Furthermore, the frequency spread of the fibres used has to be small to allow for high $Q$ notch filtering in the length control servo. The requirements for the violin modes of the two GEO 600 inboard suspensions are $Q<3 \times 10^{6}$ for the fundamental and $Q<2 \times 10^{6}$ for the first harmonic mode, respectively. The frequency spread should not exceed $10 \%$ within one mode. To accomplish that, two sections of the fibres were coated with amorphous Teflon. By applying the coating, the $Q$ of the relevant modes can be degraded to the desired values and furthermore, the frequencies of these modes can be tuned almost independently with a good accuracy over a wide range. After welding the fibres in the monolithic suspension, a corrective coating was applied to some fibres, to compensate for the frequency spread due to the tension spread of the four fibres within a suspension. We present the method and the results achieved.
\end{abstract}

PACS number: 04.80.Nn 


\section{Introduction}

Reducing the thermal noise of the suspended test masses is a key issue for the advanced interferometric gravitational-wave detectors. Hence, worldwide research is currently being carried out to build mirror suspensions with high mechanical quality factors $Q$ [1-3]. High $Q$ suspensions, such as those planned for the second generation detectors are already installed in the GEO 600 detector [4-6]. The interferometer mirrors and the beamsplitter are suspended monolithically by fused silica fibres. The transversal mechanical eigenmodes of the fibres, the so-called 'violin modes', are coupled to the motion of the test masses and may thereby cause instability of the interferometer length control servo. Due to the long ring-down times of the high $Q$ violin modes, the interferometer can have long down times. In order to avoid any excitation of the violin modes, the unity gain frequency of the length control servo is limited. To keep the servo stable and to allow further increase of the unity gain frequency in the future, we damped the violin mode $Q$. Based on thermal noise considerations, the damping was done in such a way that the pendulum $Q$ itself would be degraded as little as possible. In the following the GEO 600 inboard-mirror suspension scheme, the damping technique and the results achieved are discussed.

\section{Inboard-mirror suspension scheme}

In GEO 600 the monolithic suspension is installed as the lowest stage of a triple pendulum suspension, which has two additional vertical cantilever-spring stages (see figure 1). The triple pendulum is supported by three stack isolators, each containing a passive and an active layer. The upper pendulum mass is suspended by two steel wires from two cantilever springs. Shadow sensors and magnet-coil actuators are located at the upper mass, in order to damp the relevant pendulum resonances in six degrees of freedom. These actuators are also used for steering the mirrors manually and for the low-frequency automatic alignment control of the cavities [7]. A further four cantilever springs are attached at the lower side of the upper mass. From these springs the intermediate mass is suspended with two steel wire loops $[4,8,9]$.

Like the mirror, the intermediate mass is made of fused silica. Standoff plates, the socalled 'ears', are attached onto flat sides of the mirror and the intermediate mass substrate by silicate bonding $[10,11]$. These ears are fused silica plates, each providing two tips to weld the fibres onto. The fibres are produced from suprasil 2 rods with $5 \mathrm{~mm}$ diameter, by a pulling machine that is controlled by a computer. As a first step a rod is heated by five hydrogen-oxygen burners arranged in a star pattern and then pulled by about $50 \mathrm{~mm}$ to produce a thinner region of about $3 \mathrm{~mm}$ diameter, the so-called 'neck'. This neck defines the shape of the tapered fibre ends. The neck is then heated again and pulled by approx. $280 \mathrm{~mm}$ to produce the fibre. The quality of the fibre is then judged by its length, vertical resonance frequency and the first two violin mode frequencies.

Due to the way the fibres are produced, their central section is slightly thicker than the outer sections. The breaking load and the dilution factor of the fibre are dominated by the thiner sections, while the violin mode frequencies also depend on the thicker central section. To produce a strong fibre with high violin modes, it is desirable to produce a homogeneous fibre. However, the homogeneity of the fibres is sufficient when the above-mentioned parameters are in the targeted region. The requirements for the fundamental violin mode frequency and the obtained fibre homogeneity are given in the following sections. Before coating the fibre, the homogeneity of the fibre's diameter over its entire length is judged by the visual inspection of the symmetry of the occurring bending radius over the fibre's length, when bending it such 


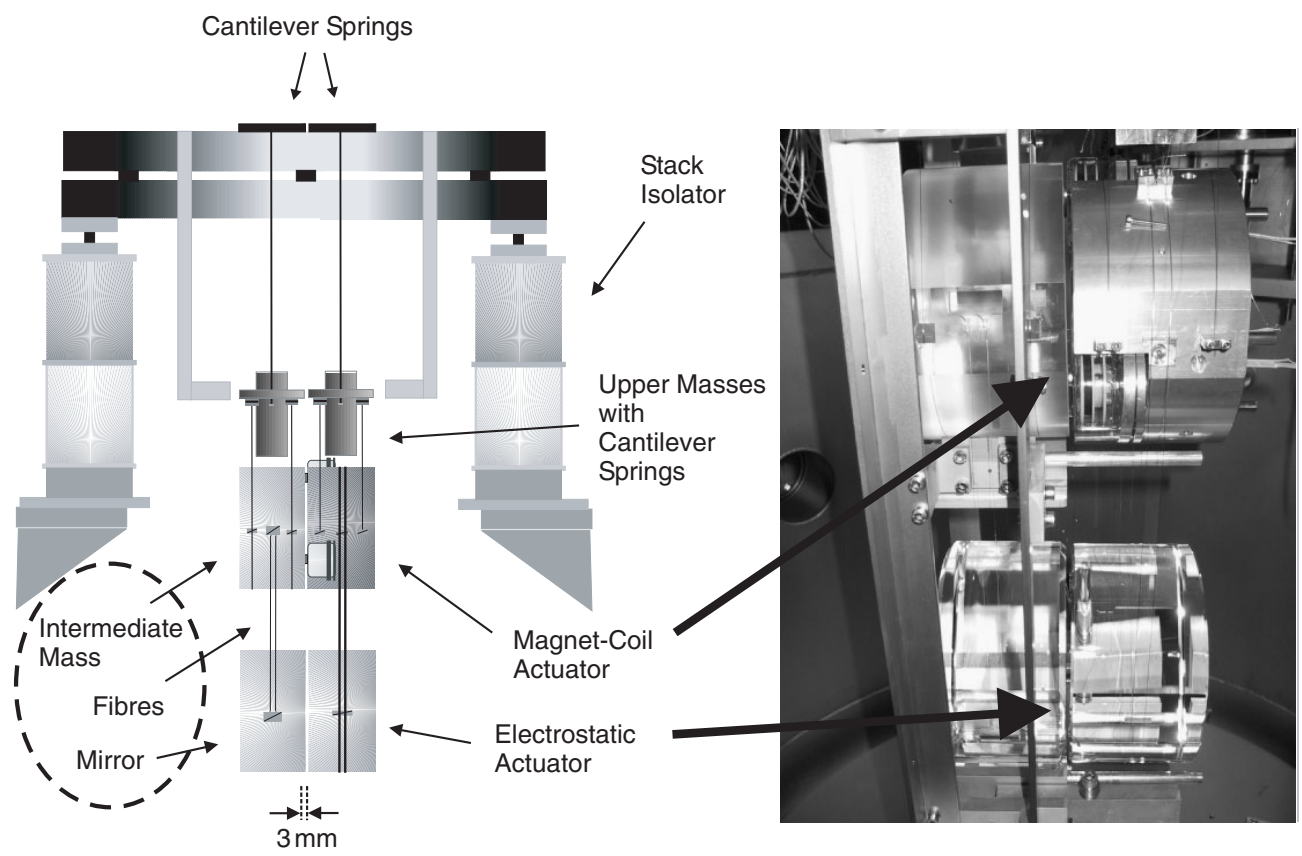

Figure 1. Left: schematic of the triple-pendulum suspension of the GEO 600 inboard interferometer mirrors. The monolithic stage is formed by the encircled constituents: the fused silica mirror is suspended with four fused-silica fibres from the fused silica intermediate mass. This monolithic stage is suspended as the third stage of the triple pendulum. Both the upper mass and the intermediate mass are suspended by steel wires. Another triple pendulum is suspended $3 \mathrm{~mm}$ behind the mirror pendulum to allow actuation on the mirror pendulum from a seismically isolated platform. For the low-frequency feedback a magnet-coil actuator is used at the intermediate mass while an electrostatic actuator allows fast feedback at the mirror itself. Right: one of the monolithic inboard-mirror suspensions with the reaction pendulum suspended behind it.

that it nearly forms an $\Omega$. The coating for the first higher order violin mode is then applied at the thiner side of the fibre.

A so-called 'cutting jig' can be set to the required length and ensures that all the fibres are cut to the same length by scratching the fibre with a glass saw at the neck and breaking the remaining part of the glass rod off. By welding the fibres with the remaining part of the neck to the tips of the ears of both masses a monolithic suspension is formed [1].

The control scheme of GEO 600 necessitates the possibility of applying length control feedback to two mirrors of the interferometer. The folding mirrors of the interferometer arms, the so-called 'far mirrors', are hanging free, while the inboard mirrors are controlled from a seismically isolated platform: a second triple pendulum, the reaction pendulum, is suspended $3 \mathrm{~mm}$ behind the mirror pendulum. The reaction pendulum contains two different actuators for the longitudinal control of the interferometer: a magnet-coil actuator at the intermediate mass, providing feedback from dc up to $10 \mathrm{~Hz}$ with a range of about $1 \mathrm{~mm}$, and an electrostatic actuator with a range of a few micrometers at the mirror itself, for the fast feedback.

\section{Design requirements}

High $Q$ suspensions normally have high $Q$ violin modes with $Q$ values exceeding $10^{8}$. The GEO 600 far-mirror monolithic suspensions, made in June 2001 with slightly damped violin 
modes, show values from $Q \approx 10^{7}$ to $Q \approx 3 \times 10^{8}$. Measurements from Caltech [12] demonstrated violin mode $Q$ of up to $5 \times 10^{8}$. Once the violin modes are excited, they cause the suspended mirror to oscillate as well. Given that the resonant enhancement of the fibres motion scales with the $Q$, the oscillation can reach a significant amplitude. Thus, the fibre motion can be driven by the feedback actuators to amplitudes that lead, via the induced mirror motion, to instabilities of the length control servo.

Even if the servo design allows a stable operation, other events could drive the violin modes to high amplitudes. Then, as the decay time of the fibre motion scales with the $Q$ also, the disturbance may last for a long time and cause problems with sensitive operation of the detector.

As described above, the interferometer length control scheme requires to feedback to the inboard suspensions, using the two actuators of the reaction pendulum. In order to avoid an excitation of the violin modes by these actuators and for the reasons discussed above, not only the length control servo must be carefully designed, but also the violin modes must fulfil certain requirements, according to their $Q$ and frequencies. At least one high $Q$ notch filter must be included in the servo design to avoid instability at the violin mode frequencies. The number and shape of the filters needed, depends significantly on the $Q$ and on the frequency spread of the violin modes. From modelling possible transfer functions of the length control servo, we derived the following requirements for the violin modes:

(i) fundamental violin mode $f_{0}: Q_{0} \leqslant 3 \times 10^{6}$,

(ii) first harmonic violin mode $f_{1}: Q_{1} \leqslant 2 \times 10^{6}$,

(iii) all frequencies within one mode $f_{i}$ (i.e., eight frequencies per mode for the two suspensions) within $\pm 5 \%$, for the first two modes $f_{0}, f_{1}$,

(iv) frequencies as high as possible $\left(f_{0} \sim 650 \mathrm{~Hz}\right)$.

Since the higher order violin modes couple less strongly to the length control servo, they do not need any extra damping. However, most of the higher order modes will be well damped by the damping of the first two modes.

\section{The $Q$-measurement setup}

Measuring high mechanical quality factors requires a low loss environment. To accomplish this we suspended a fibre with its nominal load of $1.4 \mathrm{~kg}$ from a $220 \mathrm{~g}$ isolation bob, as shown in figure 2. With a length of $280 \mathrm{~mm}$ and a diameter of approx. $220 \mu \mathrm{m}$ this fibre had the parameters later used for the inboard suspension. A diameter of about $220 \mu \mathrm{m}$ was chosen as a trade-off between the two goals of having high violin mode frequencies and having a sufficient safety margin in terms of breaking stress.

The violin modes were exited by an electrostatic actuator, consisting of a cylindrical Macor block of $25 \mathrm{~mm}$ length and $8 \mathrm{~mm}$ diameter, with a bifilar thread machined around it. In the two parallel grooves of this double thread two copper wires are spooled around the cylinder, one of them grounded while the other is charged with amplitude-modulated high voltage. The modulation frequency was supplied by a spectrum analyser and set manually without closing a feedback loop.

A HeNe laser and a split-photodiode were used to read out the fibre motion. The enlarged laser beam was adjusted to illuminate the fibre through a view port and was then sensed with the split-photodiode such that the shadow of the fibre covered a part of both independent sections of the diode. With this setup we were able to enhance the violin modes to about $80 \mathrm{~dB}$ above the noise level of the readout system. 


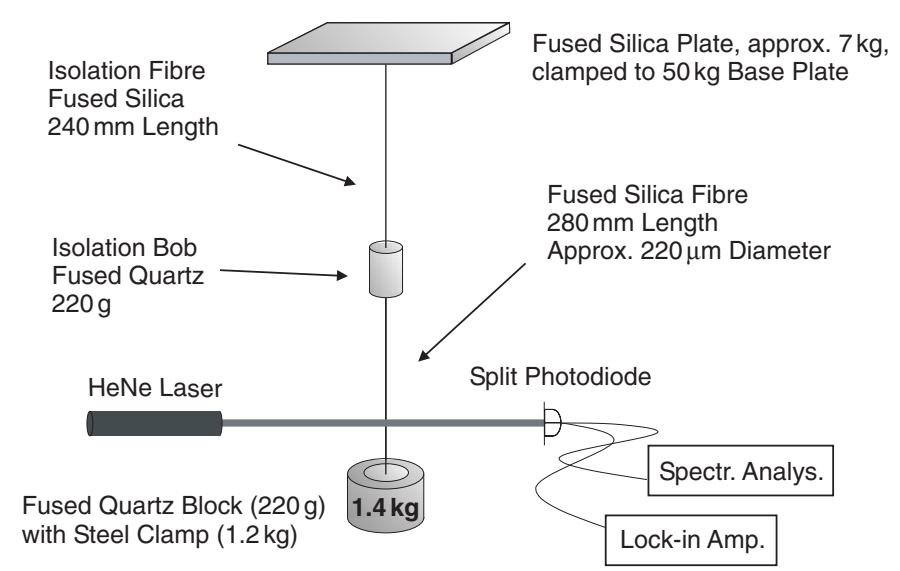

Figure 2. Setup for the $Q$ measurements: a fused silica plate is rigidly clamped to an aluminium plate that is mounted on three sturdy posts. A first fused silica suspension stage contains an isolating bob of $220 \mathrm{~g}$ of fused quartz. From that bob we suspended a fibre having the parameters (i.e., length, diameter, bounce-mode frequency) needed for the GEO suspension under the nominal load of $1.4 \mathrm{~kg}$. We used a HeNe laser and a split photodiode for the readout of the fibre motion, while we used an electrostatic actuator (not shown here) to drive the fibre resonances.

The differential signal of the split photodiode was fed to a lock-in amplifier and the amplifier signal was recorded with a computer based data acquisition system. Finally, the $Q$ of the sample was calculated from the ring-down time, using

$$
Q_{i}=\pi f_{i} \tau
$$

where $f_{i}$ is the resonance frequency of the $i$ th eigenmode and $\tau$ is the time measured for decay to $1 / e$ amplitude.

\section{The damping method}

The idea behind the damping method is simply to apply some 'lossy' material to sections of the fibre that will affect the relevant violin mode $Q$, but not the $Q$ of the pendulum itself. These sections should have a reasonable bending radius for the violin modes but not for the pendulum mode. Except for the area around the attachment points, the smallest radius of curvature of an oscillating fibre is in the fibres centre for the fundamental eigenmode $f_{0}$, and at about one fourth of the fibres length for the first harmonic mode $f_{1}$, respectively. Except for the attachment point, an ideal fibre has an infinite radius of curvature over its entire length for the pendulum mode. The bending of a real fibre due to the pendulum mode decreases with the distance $x$ from the break-off point (which is in fact not a point but a region, that is several $\mathrm{mm}$ long in the GEO 600 case), following an exponential law proportional to $\frac{\mathrm{d}^{2}}{\mathrm{~d} x^{2}} \mathrm{e}^{\frac{-x}{\lambda}}$, with $\lambda=\sqrt{\frac{E_{\mathrm{S}} I}{T}} . E_{\mathrm{S}}$ is the Young modulus of fused silica $\left(E_{\mathrm{S}}=7 \times 10^{10} \mathrm{~Pa}\right), T$ is the tension and $I=\frac{\pi}{4} R^{4}$ is the bending moment of inertia of the fibre. With $\lambda$ being in the order of about $10^{-3} \mathrm{~m}$, the bending decreases rapidly with increasing distance from the break-off point. Thus, the fibre can be regarded as practically straight at the coated sections and the degradation of the pendulum mode $Q$ is negligible. For the two first violin modes instead the two patches are located where the bending has a maximum. 


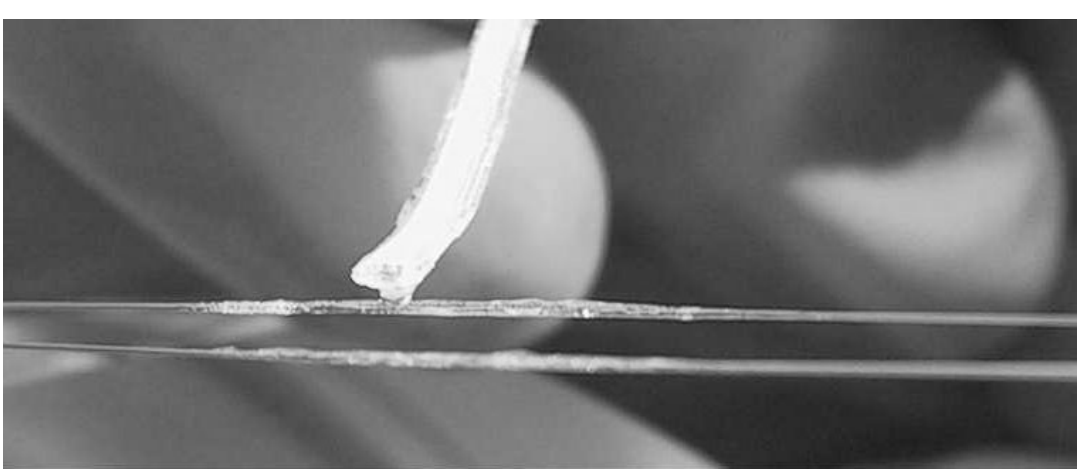

Figure 3. Close-up view of the coating process: a drop of the dissolved Teflon is applied with a Teflon stick, without touching the fibre. This picture shows the 'in situ' application of the corrective coating onto one fibre of a monolithic suspension.

The lossy material was chosen to be amorphous Teflon, because we proved earlier that it does not degrade the fibre's strength [1] and that it is ultra-high vacuum compatible. The Teflon is dissolved in flourinert and its viscosity can be set by the amount of solvent added. To bring the Teflon onto the fibres, a little stick of Teflon is used to pick up a drop of the dissolved material. Only this drop is brought into contact with the fibre, because the fibres must not be touched with anything else. The drop is then guided back and forth until it is almost completely applied to the fibre. This is done several times, such that a coating is formed (see figure 3). The solvent has a high vapour pressure and hence it almost completely evaporates within half an hour, leaving the Teflon coating on the fibre. By measuring fibres with different coating lengths and diameters, it was proved that the damping effect of the applied Teflon scales much more strongly with the coating diameter then with the coating length. This clearly indicates that the damping effect comes not from the rubbing of the fibre with the Teflon, but from the loss of the Teflon itself. The downwards shifting of the violin mode frequencies scales simply with the added mass, weighted with the coating position: since the first harmonic violin mode has a node at the centre of the fibre, the fundamental mode can be tuned with almost no influence on the first harmonic. Thus, by varying the position, length and thickness of the coating the frequencies and $Q$ of the first two violin modes can be separately tuned.

\subsection{Influence on the vertical mode}

Measurements showed that the vertical mode frequency is not affected by the damping of the first two violin modes. However, the $Q$ of the vertical mode is affected by the damping of the violin modes and hence, an estimation of the introduced thermal noise has to be performed. An estimation for the $Q$ of the vertical mode can be derived from the loss of the violin modes. It is straightforward to approximate the energy stored in the violin mode oscillation from the kinetic energy of the fibre at the zero crossing to be

$$
E_{\text {stored }} \simeq \frac{1}{2} S \rho A^{2} \omega^{2} \int_{0}^{L} \sin ^{2}\left(\frac{(n+1) \pi}{L} z\right) \mathrm{d} z=\frac{1}{4} S \rho L A^{2} \omega^{2}
$$

where $L$ is the fibre length, $n$ is the violin mode number, $A$ is the oscillation amplitude, $S$ is the cross-section area and $\rho$ is the material mass density. The energy $E_{\text {coating }}$ stored in the coating can be approximated by 


$$
\begin{aligned}
E_{\text {coating }} & =\frac{1}{2} \int_{\bar{z}-\frac{l}{2}}^{\bar{z}+\frac{l}{2}} I E_{C}\left(\frac{\mathrm{d}^{2} Y(z)}{\mathrm{d} z^{2}}\right)^{2} \mathrm{~d} z \\
& \simeq \frac{1}{2} I E_{C} A^{2}\left(\frac{(n+1) \pi}{L}\right)^{4} l \sin ^{2}\left(\frac{(n+1) \pi}{L} \bar{z}\right)
\end{aligned}
$$

where $E_{C}$ is the Young modulus of the coating, $\bar{z}$ is the centre of the coated section, $l$ is the coating length of this section, $Y(z)$ is the transversal displacement of the fibre with respect to the rest position, and

$$
I=\frac{\pi}{4}\left((R+h)^{4}-R^{4}\right)
$$

is the bending moment of inertia of the coating, where $R$ gives the fibre radius and $h$ is the thickness of the coating. In equation (3) it was assumed that $Y \simeq A \sin ^{2}\left(\frac{(n+1) \pi}{L} z\right)$ and that $l \ll \frac{L}{2(n+1)}$. Under the assumption that the dissipation is mainly caused by the loss $\phi_{\mathrm{C}}$ of the damping material, the energy dissipated per cycle is given by

$$
E_{\text {dissipated }} \simeq 2 \pi \phi_{\mathrm{C}} E_{\text {coating }} \text {. }
$$

For a fibre with two coated sections of different thicknesses at sections of the fibre with different radii, the $Q$ of the $n$th violin mode is, still under the above assumption, given by

$$
Q_{n}=2 \pi \frac{E_{\text {stored }}}{E_{\text {dissipated }}} \simeq \frac{1}{2} \frac{\rho S L \omega_{n}^{2}}{\left(\frac{(n+1) \pi}{L}\right)^{4} E_{\mathrm{C}} \phi_{\mathrm{C}} \sum_{i=1}^{2} I_{i} l_{i} \sin ^{2}\left(\frac{(n+1) \pi}{L} \bar{z}_{i}\right)} .
$$

Equation (6) has been used to work out the imaginary part of the Young modulus of Teflon from the measurements of the violin mode $Q$, to be about $E_{\mathrm{C}} \phi_{\mathrm{C}} \simeq 3 \times 10^{8} \mathrm{~Pa}$. This is a fundamental parameter for the approximation of the loss of the vertical mode:

$$
\phi_{\mathrm{vert}} \simeq \phi_{\mathrm{S}}+\frac{E_{\mathrm{C}} \phi_{\mathrm{C}}}{E_{\mathrm{S}}} \frac{l}{L}\left[\left(\frac{R+h}{R}\right)^{2}-1\right]
$$

where $\phi_{\mathrm{S}}$ is the loss factor of fused silica $\left(\phi_{\mathrm{S}} \in\left[10^{-7}, 10^{-6}\right]\right)$, the $Q$ of the vertical mode can be approximated to be degraded to about $Q_{\text {vert }} \simeq 10^{3}$.

The resulting introduced thermal noise above $50 \mathrm{~Hz}$ is well below the internal thermal noise of the GEO 600 test masses and the predicted thermo-refractive noise. Between $50 \mathrm{~Hz}$ and $20 \mathrm{~Hz}$ it is still well below the thermo-refractive noise. Thus, the calculations indicate that the thermal noise induced by this 'parasitic' damping of the vertical mode will not affect the sensitivity of GEO 600 in the most sensitive frequency band. However, measurements of the degradation of the vertical mode $Q$ could not be done with our setup and hence, still need to be performed.

\section{Measurements in the lab}

Using the $Q$-measurement setup and the damping method described above, we prepared a fibre with the parameters needed for the inboard suspension (see section 3). As a first step the fibre was welded in the monolithic double pendulum as the second stage. The $Q$ and frequencies of the first two modes were measured, and then a small amount of the Teflon was applied at two sections of the fibre. This was done by bending the upper stage of the double pendulum by $90^{\circ}$ into a horizontal position and holding the lower fibre straight. Measuring and remeasuring the $Q$ and frequencies with gradual application of more Teflon eventually led to a fibre with the properties aimed for (see figure 4). The fibre showed quality factors of $Q_{0}=2 \times 10^{6}$ and $Q_{1}=5 \times 10^{5}$ at $f_{0}=664 \mathrm{~Hz}$ and $f_{1}=1314 \mathrm{~Hz}$ for the fundamental and the first harmonic mode, respectively. 


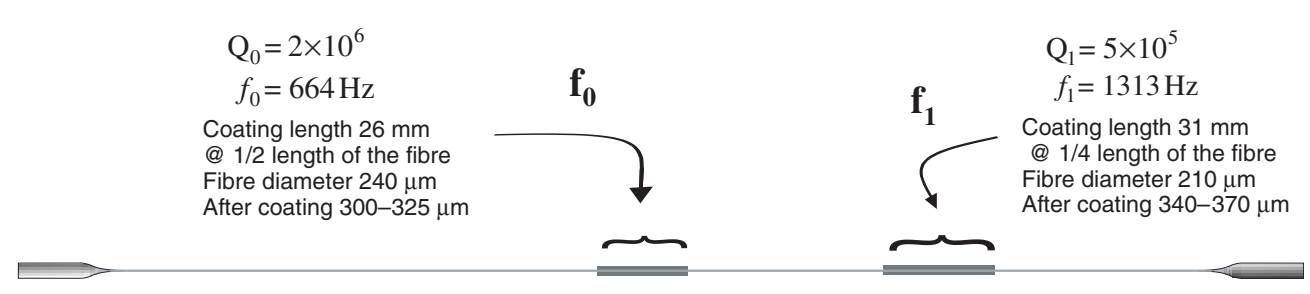

Figure 4. The reference fibre: two sections of the fibre were coated in an iterative process until the $Q$ of the first two violin modes showed tolerable values. The fibre length is $284 \mathrm{~mm}$, the diameter $210-240 \mu \mathrm{m}$. Before coating the frequencies were $664 \mathrm{~Hz}$ for the fundamental and $1313 \mathrm{~Hz}$ for the first higher order mode, respectively.

Table 1. Two examples of the frequency tuning procedure. The coating was applied in an iteration to meet the requirements. In the first example the fundamental violin mode $f_{0}$ was still too high when the first harmonic $f_{1}$ was already in the target region. In the second example the fundamental mode $f_{0}$ of a different fibre was already in the target region when the first harmonic mode $f_{1}$ was still too high. The achieved results after the second correction demonstrate the possibility of tuning the modes independently.

\begin{tabular}{lcccc}
\hline & Uncoated & After coating & $\begin{array}{l}\text { After corrective } \\
\text { coating }\end{array}$ & $\begin{array}{l}\text { After second } \\
\text { correction coating }\end{array}$ \\
\hline$f_{0}(\mathrm{~Hz})$ & 741 & 691 & 678 & 665 \\
$f_{1}(\mathrm{~Hz})$ & 1415 & 1325 & 1316 & 1316 \\
$f_{0}(\mathrm{~Hz})$ & 724 & 676 & 662 & 660 \\
$f_{1}(\mathrm{~Hz})$ & 1490 & 1380 & 1353 & 1306 \\
\hline
\end{tabular}

This fibre was taken as a reference for the production of the required number of fibres for the two mirror suspensions with as similar as possible properties. Since it is not possible to unweld a fibre and then use it for a further suspension, it is not possible to measure the violin mode $Q$ before the fibre is welded in and suspended in vacuum. However, it is possible to measure the frequencies and therefore to reproduce fibres with a similar down shift in frequency for the first two modes. If the coatings and fibres are of a similar geometry then comparable reductions in $Q$ are also to be expected.

As already described, it is possible to tune the fundamental mode $f_{0}$ almost without influencing the first higher-order mode $f_{1}$, since the latter one has a node at the centre of the fibre. Tuning the first harmonic mode $f_{1}$ however influences the fundamental mode $f_{0}$, but much less than the first harmonic itself. Two examples of the tuning procedure, demonstrating the independence between the two modes are given in table 1 .

\section{Measurements at GEO 600}

Before coating the fibres, a frequency $f_{0}=740 \mathrm{~Hz} \pm 3.5 \%$ and $f_{1}=1460 \mathrm{~Hz} \pm 4.2 \%$ was obtained for the production of 30 fibres. By coating the fibres, the frequencies of 20 of the 30 fibres were brought to $f_{0}=663 \mathrm{~Hz} \pm 0.48 \%$ and $f_{1}=1314 \mathrm{~Hz} \pm 0.86 \%$. The fact that the centre frequencies of this set of coated fibres were only $1 \mathrm{~Hz}$ away from the target frequencies for both modes, shows the accuracy of the method.

Unfortunately, the frequency spread is very likely to be enlarged by welding and suspending the monolithic stage. This is caused by the fact that it is not possible to cut the four fibres, required for one suspension, exactly to the same length and weld them under 
exactly the same tension. Welding slightly unequally with respect to the centre of mass of the mirror also causes a different tension of the fibres when the monolithic stage is suspended. Therefore we remeasured the frequencies after the fibres were welded into the monolithic suspensions, with the lower mass suspended as will be described in the next paragraph. The spread of the fundamental mode for both suspensions was $\pm 3.35 \%$ after welding and suspending in a test setup. We applied a corrective coating onto two fibres to further reduce the spread, since an additional enlargement of the spread can be expected from mounting the monolithic stages in the triple-pendulum suspensions. The origin of this spread is the coupling of both pitch and roll alignment to the fibre tensions, via a shift of the centre of mass (COM) of the lower mass. The COM is shifted because the bending point of the fibres is above the COM for stability reasons and, hence, the COM lies not on the axes of roll or pitch motion. This coupling causes any difference between the final aligned suspension and the test setup to change the fibre tensions.

To weld the fibres to the ears of the mirror and the intermediate mass, we use a catcher that holds the two masses. The catcher gives the right distance between the masses and allows the longitudinal alignment along the normal to the optical surface. It also allows the alignment of the masses with respect to roll around the normal to the optical surface. In the correct position, the masses are fixed by clamps. Directly after the welding the monolithic stage can be suspended inside the catcher to confirm the strength of the new monolithic stage, and to measure the violin mode frequencies. After the measurements the monolithic stage can be clamped back into the catcher. The catcher allows us to safely rotate the clamped suspension around all axes and to move it over long distances (e.g., from one end station of GEO 600 to the other). Hence, it can be laid on its sides, providing access to the fibres for applying an additional amount of Teflon to reduce the frequencies if required. This was done for two of the eight fibres and thereby the frequency spread of the fundamental mode of both suspensions was reduced to $\pm 2.65 \%$.

After installing the two monolithic stages as the last stages of the two inboard triple pendulums and evacuating the system, we excited the violin modes with the suspended electrostatic actuators. The sinusoidal modulation frequencies were provided by a waveform generator and were adjusted manually to the violin mode frequencies. An error-point spectrum of the length control servo was analysed with a spectrum analyser to find the frequencies and to adjust the modulation frequencies. The spectrum was then recorded with the data acquisition system of GEO 600 [13]. A snapshot of the error-point spectrum is displayed in figure 5. The resulting frequency spread of the violin modes of the two inboard suspensions is $f_{0}=645.5 \mathrm{~Hz} \pm 3.86 \%$ and $1287 \mathrm{~Hz} \pm 4.86 \%$.

The $Q$ of the first two modes $f_{0}$ and $f_{1}$ were derived from the ring-down times to be $Q_{0}=5 \times 10^{5}-2.8 \times 10^{6}$ and $Q_{1}=5.4 \times 10^{5}-1 \times 10^{6}$.

With these frequencies, frequency spread and $Q$, the monolithic suspensions well meet the requirements set by the length control servo demands.

\section{Future perspectives}

The successful installation of the GEO 600 inboard suspensions demonstrated that it is possible to model the $Q$ and frequencies of the suspension fibres to values, tolerable for the length control servos. Calculations indicate that the additional vertical thermal noise introduced thereby will not affect the sensitivity of GEO 600 in the most sensitive frequency range.

A 'side' experiment showed that it is even possible to apply a corrective coating in vivo to a suspended fibre. That allows a correction of the additional frequency spread, introduced 


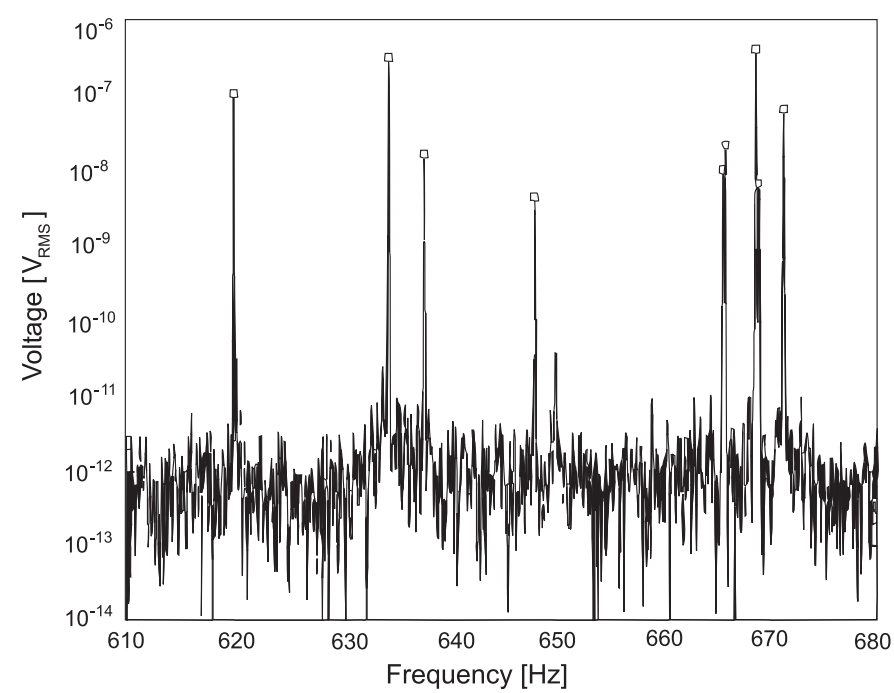

Figure 5. Error-point spectrum of the length control servo of GEO 600: Nine violin mode peaks are resolved; the $650 \mathrm{~Hz}$ peak belongs to the power line. The eight fibres of the two inboard suspensions have, since the fibre cross sections are not perfectly round but slightly elliptical, in principle 16 violin modes. However, the frequency separation of the orthogonal modes due to this asymmetry is in most cases below the measurement resolution used. The violin modes were driven at their resonance frequencies with the electro-static actuator with a sinusoidal voltage, supplied by a signal generator.

by the installation of the monolithic stage as the last stage of a multiple pendulum. In this experiment $f_{0}$ of an already coated fibre was tuned in three steps further down by $8 \mathrm{~Hz}$.

In a second side experiment a fibre was coated over almost its entire length, with a very small amount of Teflon. The fibre treated in this way shows a much greater tolerance to the environment. It is possible to touch the loaded fibre with a glass rod without breaking the fibre. In contrast to an uncoated fibre the fibre strength seems not to be degraded by the application of a drop of water directly onto this fibre. The violin modes shifted downwards by about $15 \mathrm{~Hz}$, which means that a degradation of the violin mode $Q$ can be expected to be only small. In contrast to that, the vertical mode $Q$ can be affected quite strongly by such a coating. However, a very thin protective coating can make the handling of these touchy fibres much easier, but further investigations need to be done, since only one fibre was treated in this way so far.

\section{Acknowledgments}

The authors would like to thank the Byer Feijer group at the Ginzton Laboratory, Stanford, for the supply of the amorphous Teflon used in their laser development programme. This work was supported by PPARC in the UK, the BMBF in Germany and the state of Lower Saxony.

\section{References}

[1] Barr B W et al 2002 Silica reaserch in Glasgow Class. Quantum Grav. 19 1655-63

[2] Amico P, Bosi L, Carbone L, Gammaitoni L, Punturo M, Travasso F and Vocca H 2002 Fused silica suspensions for the VIRGO optics: status and perspectives Class. Quantum Grav. 19 1669-75 
[3] Ju L, Blair D G, Bilenko J and Padget D 2002 Low loss niobium flexure suspension systems Class. Quantum Grav. 19 1703-9

[4] Goßler S et al 2002 The modecleaner system and suspension aspects of GEO 600 Class. Quantum Grav. 19 $1835-43$

[5] Willke B et al 2002 The GEO 600 gravitational wave detector Class. Quantum Grav. 19 1377-87

[6] Smith J R, Cagnoli C, Crooks D R M, Goßler S, Lück H, Rowan S, Hough J and Danzmann K 2003 Class. Quantum Grav. 21 S1091

[7] Grote $\mathrm{H}$ et al 2002 The automatic alignment system of GEO 600 Class. Quantum Grav. 19 1849-57

[8] Plissi M V, Strain K A, Torrie C I, Robertson N A, Killbourne S, Rowan S, Twyford S M, Ward H, Skeldon K D and Hough J 1998 Aspects of the suspension system for GEO 600 Rev. Sci. Instrum. 69 3055-61

[9] Plissi M V, Torrie C I, Husman M E, Robertson N A, Strain K A, Ward H, Lück H and Hough J 2000 GEO 600 triple pendulum suspension system: seismic isolation and control Rev. Sci. Instrum. 71 2539-45

[10] Sneddon P H, Bull S, Cagnoli G, Crooks D R M, Elliffe E J, Faller J E, Fejer M M, Hough J and Rowan S 2003 The intrinsic mechanical loss factor of hxdroxy-catalysis bonds for use in the mirror suspensions of gravitational wave detectors Class. Quantum Grav. 20 5025-37

[11] Smith J R et al Class. Quantum Grav. at press

[12] Willems P et al 2002 Investigations of the dynamics and mechanical dissipation of a fused silica suspension Phys. Lett. A 297 37-48

[13] Kötter K et al 2002 Data acquisition and detector characterization of GEO 600 Class. Quantum Grav. 19 1399-409 